Brief Report

\title{
Competing Fluid Forces Control Endothelial Sprouting in a 3-D Microfluidic Vessel Bifurcation Model
}

\author{
Ehsan Akbari ${ }^{1}{ }^{\mathbb{D}}$, Griffin B. Spychalski ${ }^{2}$, Kaushik K. Rangharajan ${ }^{1}$, Shaurya Prakash ${ }^{1}$ \\ and Jonathan W. Song 1,3,*iD \\ 1 Department of Mechanical and Aerospace Engineering, The Ohio State University, \\ Columbus, $\mathrm{OH} 43210$, USA \\ 2 Department of Biomedical Engineering, The Ohio State University, Columbus, OH 43210, USA \\ 3 Comprehensive Cancer Center, The Ohio State University, Columbus, OH 43210, USA \\ * Correspondence: song.1069@osu.edu; Tel.: +1-614-292-8519
}

Received: 5 June 2019; Accepted: 2 July 2019; Published: 4 July 2019

\begin{abstract}
Sprouting angiogenesis—the infiltration and extension of endothelial cells from pre-existing blood vessels-helps orchestrate vascular growth and remodeling. It is now agreed that fluid forces, such as laminar shear stress due to unidirectional flow in straight vessel segments, are important regulators of angiogenesis. However, regulation of angiogenesis by the different flow dynamics that arise due to vessel branching, such as impinging flow stagnation at the base of a bifurcating vessel, are not well understood. Here we used a recently developed 3-D microfluidic model to investigate the role of the flow conditions that occur due to vessel bifurcations on endothelial sprouting. We observed that bifurcating fluid flow located at the vessel bifurcation point suppresses the formation of angiogenic sprouts. Similarly, laminar shear stress at a magnitude of $\sim 3 \mathrm{dyn} / \mathrm{cm}^{2}$ applied in the branched vessels downstream of the bifurcation point, inhibited the formation of angiogenic sprouts. In contrast, co-application of $\sim 1 \mu \mathrm{m} / \mathrm{s}$ average transvascular flow across the endothelial monolayer with laminar shear stress induced the formation of angiogenic sprouts. These results suggest that transvascular flow imparts a competing effect against bifurcating fluid flow and laminar shear stress in regulating endothelial sprouting. To our knowledge, these findings are the first report on the stabilizing role of bifurcating fluid flow on endothelial sprouting. These results also demonstrate the importance of local flow dynamics due to branched vessel geometry in determining the location of sprouting angiogenesis.
\end{abstract}

Keywords: angiogenesis; shear stress; biomechanics; vessel branching

\section{Introduction}

Blood vessels comprise a hierarchical network that transports oxygen and nutrients throughout the body [1]. Expansion of this network occurs by angiogenesis, where endothelial cells (ECs) that line the inner surface of all blood vessels, sprout and branch to support tissue nourishment and growth [2,3]. Angiogenesis is necessary to help repair injured tissue [4], while uncontrolled angiogenesis is a prominent characteristic of rapidly growing solid tumors [5]. Therefore, the growth and remodeling of blood vessels by angiogenesis is critical throughout physiology [6,7], and increasing our fundamental understanding of angiogenesis is necessary to improve therapeutic strategies used for regenerative and cardiovascular medicine, and cancer therapy.

Research on angiogenesis has traditionally focused on identifying biochemical signaling factors that regulate EC function [8]. Yet in physiology, blood vessels are continuously exposed to fluid mechanical forces due to blood flow, including shear stress tangential to the endothelium, and normal flow across the vessel wall. Emerging research has highlighted the importance of the forces 
generated by blood flow in potently influencing the angiogenic process. These studies in the in vitro setting have been buoyed largely by the advancements in microfabrication techniques that enable the development of perfusable models that integrate 3-D tissue scaffolds for investigating angiogenesis in response to controlled fluid forces $[9,10]$. For instance, the application of $3 \mathrm{dyn} / \mathrm{cm}^{2}$ laminar shear stress (LSS) was shown to suppress endothelial sprouting induced by vascular endothelial growth factor (VEGF) [11], while LSS values greater than $10 \mathrm{dyn} / \mathrm{cm}^{2}$ have been shown to induce angiogenic sprouting [12]. Furthermore, previous studies have shown the pro-angiogenic role of transvascular flow (TVF) that is driven by a transmural pressure difference between the vasculature and the adjacent interstitium [11-13].

Endothelial sprouting has predominantly been examined in in vitro microfabricated devices with straight blood vessel models. However, the in vivo vasculature consists of a hierarchy of branching structures that generates bifurcating fluid flow (BFF) at the base of vessel bifurcations. BFF can be characterized by local stagnation pressure imparted normal to the endothelium and near-zero average shear stress, distinguishing BFF from previously studied hemodynamic factors (i.e., LSS and TVF). Recently, we reported an in vitro microfluidic model of a branching vessel that enables measurement of vascular permeability under application of BFF, LSS, and TVF [14] while also presenting an interface between fluid flow, ECs, and 3-D extracellular matrix (ECM). The findings from this study demonstrated the vascular permeability outcomes in response to the local flow dynamics at specific locations along the bifurcating vessel structure. However, the role of BFF in co-regulating endothelial sprouting has not been reported previously. Since elevated vascular permeability typically coincides with increased angiogenesis [15], the purpose of this paper was to investigate endothelial sprouting due to the flow dynamics produced by branching vessel geometry.

Here we report that application of BFF with $\sim 38 \mathrm{dyn} / \mathrm{cm}^{2}$ stagnation pressure at the vessel bifurcation point (BP) and $\sim 3 \mathrm{dyn} / \mathrm{cm}^{2}$ LSS in each branched vessel (BV) inhibits the formation of angiogenic sprouts. Furthermore, co-application of TVF with BFF and LSS induces the formation of angiogenic sprouts, thereby suggesting the presence of competing effects between TVF with BFF and LSS in regulating endothelial sprouting in a vessel bifurcation model. This work presents the first quantitative report on the stabilizing role of BFF for inhibiting endothelial sprouting. Moreover, these results demonstrate that sprout location can be determined by the local flow dynamics due to vessel bifurcations. Consequently, the findings reported here advance the current understanding of the fluid mechanical regulators of angiogenesis.

\section{Materials and Methods}

\subsection{Microfluidic Model of Vessel Bifurcation}

A microfluidic platform was developed as a 3-dimensional (3-D) in vitro analogue of a bifurcating vessel as previously described [14] (Figure 1A,B). The microchannels were fabricated by soft lithography of polydimethylsiloxane (PDMS), as reported previously [14]. Upon seeding, the microchannels were fully lined with mouse aortic endothelial cells (MAECs) to form an in vitro vessel analogue (Figure 1D). An important feature of the microfluidic model is a 3-D ECM compartment comprised of a mixture of collagen gel $(3 \mathrm{mg} / \mathrm{mL}$ rat tail-type I) and fibronectin $(10 \mu \mathrm{g} / \mathrm{mL})$ that was positioned between the two parallel BV regions and intersects the base of the branching vessel or the BP (Figure 1D). Located at the $\mathrm{BP}$ and the two BVs are $100 \mu \mathrm{m}$ wide openings (referred to as apertures) between two PDMS posts that help contain the pre-polymerized collagen gel solution in the 3-D ECM compartment [16]. These apertures enabled direct contact of the MAEC monolayer with the supporting collagen ECM where sprouts can infiltrate (Figure 1E). 

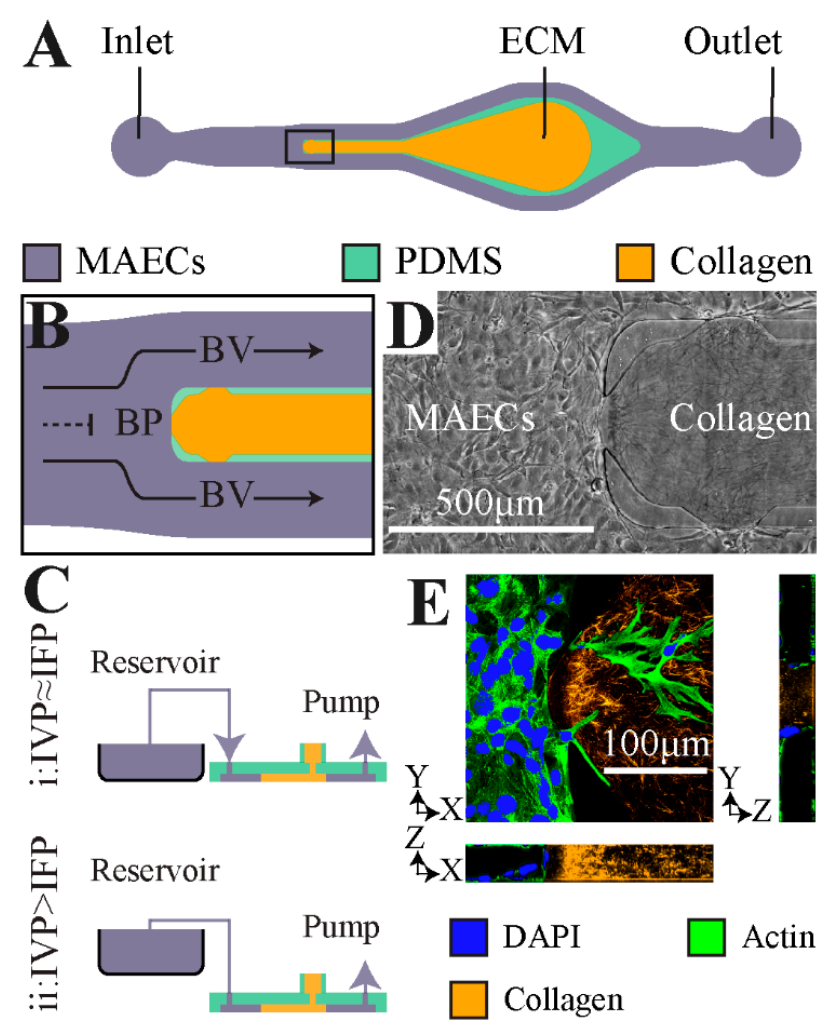

Figure 1. Schematic and characterization of the in vitro microfluidic device reported previously [14] used here for examination of sprouting angiogenesis. (A) Top-view for the microfluidic device. (B) Schematic showing a close-up for the local fluid flow at the bifurcation point (BP) aperture and the flow along apertures in the branched vessel (BV). (C) The schematic of the approach implemented to control the difference between intravascular pressure (IVP) and the interstitial fluid pressure (IFP) through controlled elevation of the reservoir. (D) A representative phase-contrast image of the BP fully lined with mouse aortic endothelial cells (MAECs) adjacent to the polymerized extracellular matrix (ECM) hydrogel. (E) A representative confocal image of the microfluidic device fully seeded with MAECs. Furthermore, the 3-D structure of the polymerized collagen matrix (orange) was resolved using total reflectance confocal imaging.

\subsection{Cell Culture and Perfusion of the Microfluidic Model}

MAECs were generously provided by the laboratory of Dr. Mike Ostrowski and cultured as previously described [17]. Briefly, MAECs were grown in DMEM-F12 cell culture media supplemented with $20 \%$ heat-inactivated fetal bovine serum, $1 \%$ penicillin-streptomycin, $10 \mathrm{U} / \mathrm{mL}$ heparin and $30 \mu \mathrm{g} / \mathrm{mL}$ endothelial cell growth supplement. The microchannels were pre-treated with fibronectin $(10 \mu \mathrm{g} / \mathrm{mL})$ for $2 \mathrm{~h}$ at $37^{\circ} \mathrm{C}$ followed by treatment with cell culture growth media overnight at $37^{\circ} \mathrm{C}$. Subsequently, MAECs at 6-10 passage number were seeded at 20,000 cells/ $\mu$ l concentration, allowed to adhere overnight at $37^{\circ} \mathrm{C}$, and grown to confluence for $24 \mathrm{~h}$. Controlled perfusion in the microfluidic model was applied using a programmable syringe pump as previously described [14]. A full-scale computational model of the microfluidic platform was developed using COMSOL Multiphysics (version 4.4) to determine: (a) the average LSS experienced by the MAECs that are adhered on the ECM at the BP and in each BV, (b) the level of TVF resulting in interstitial flow based on the measured hydraulic permeability of the ECM and the endothelial cell monolayer [14].

\subsection{Immunofluorescence}

Following treatment with each experimental condition, the microdevices were flushed 3 times with phosphate buffered saline (PBS) and fixed using 3\% paraformaldehyde for $30 \mathrm{~min}$ at room temperature. Next, the devices were flushed 3 times with PBS and incubated with the blocking buffer (5\% donkey 
serum with $0.1 \%$ Triton $X-100$ in PBS) for 1 hour at room temperature. The devices were then flushed 3 times with PBS and incubated with phalloidin (solution made by diluting the stock by 1:20 in PBS with $10 \%$ blocking buffer) for $30 \mathrm{~min}$ at room temperature to stain for actin. Next, the devices were flushed 3 times with PBS and incubated with DAPI (solution made by diluting the stock by 1:1000 in double distilled water) for $5 \mathrm{~min}$ at room temperature. Finally, the devices were flushed 3 times with PBS prior to epifluorescence imaging (TS-100, Nikon).

\subsection{Quantification of Increase in Sprouting Area}

Sprouting area was quantified using NIH ImageJ. A user-defined region of interest was defined for each aperture (i.e., BP or BV) and the total area covered by sprouting MAECs was quantified. The total sprouting area at $72 \mathrm{~h}$ after seeding was subtracted by the sprouting area at $24 \mathrm{~h}$ after seeding to report the total increase in sprouting area over $48 \mathrm{~h}$.

\subsection{Quantification of Sprout Elongation and Alignment}

The morphological responses of MAEC sprouting were quantified using an elongation index and angle of orientation, which are commonly used parameters that quantify the extent that cells elongate and align in the direction of flow, respectively [18]. The elongation index for each sprouting MAEC was defined by the major axis (X) and minor axis (Y) of an ellipse fit to the cell area using MATLAB (Equation (1)) [19]. An elongation index of 0 represents a cell fit perfectly to a circle (non-elongated), and elongation index of 1 represents a cell fit perfectly to a line (fully elongated).

$$
\text { Elongation Index }=\frac{X-Y}{X+Y}
$$

The angle of orientation between each sprouting MAEC and interstitial flow was defined by the absolute angle between the major axis of the ellipse fit to the cell area and the interstitial flow vector at the cell area centroid. An angle of orientation of $0^{\circ}$ is a cell aligned perfectly with the direction of interstitial flow and $90^{\circ}$ is a cell aligned perpendicular to the direction of interstitial flow.

\subsection{Statistical Analysis}

Each experimental test condition was conducted at least in triplicate to report statistical analysis. The values reported for the sprouting area represent the average \pm standard error of the mean. Two-sided Student's t-test was used to report statistical significance between each two pair of test conditions. The following symbols were used to report statistical significance: * indicates $p$-value $<0.05$, *** indicates $p$-value $<0.001$.

\section{Results}

\subsection{Bifurcating Fluid Flow (BFF) and Laminar Shear Stress (LSS) Attenuate Endothelial Sprouting}

The branching vessel geometry of our 3-D microfluidic model enables evaluation of angiogenic sprouting in response to BFF and LSS within the same fluidic circuit. In addition, our microfluidic model enables control of TVF levels independent of the perfusion rate, thereby enabling us to decouple the effects of TVF on endothelial sprouting from the effects of BFF and LSS. This capability is achieved by controlling the pressure difference via a static pressure head column between the intravascular pressure (IVP) and interstitial fluid pressure (IFP) domains (Figure 1C). Consequently, TVF can be either induced or restricted based on whether there is a difference in IVP and IFP [14]. When IVP is equal to IFP (IVP = IFP), TVF is $\sim 0$ (Figure 2A). Therefore, under these experimental conditions, sprouting angiogenesis responses were due to BFF and LSS and in the absence of TVF. When IVP is greater than IFP (IVP > IFP), a $1.5 \mathrm{~cm} \mathrm{H} \mathrm{H}_{2} \mathrm{O}$ hydrostatic pressure difference $\left(\sim 147 \mathrm{~Pa}\right.$ or $\left.\sim 1470 \mathrm{dyn} / \mathrm{cm}^{2}\right)$ results in a TVF of $\sim 1 \mu \mathrm{m} / \mathrm{s}$ oriented from the MAEC-lined intravascular region, across the endothelium, and into the interstitial ECM compartment (Figure 2A). TVF is a form of interstitial flow across the 
blood vessel wall [20], and in normal physiology, interstitial flow is thought to be on the order of 0.1-1 $\mu \mathrm{m} / \mathrm{s}$ [21,22]. In our microfluidic model, introduction of $\sim 147 \mathrm{~Pa}$ pressure head resulted in an average interstitial flow of $0.75 \mu \mathrm{m} / \mathrm{s}$. Therefore, under these conditions, sprouting angiogenesis was evaluated in response to co-application of BFF and LSS alongside TVF.
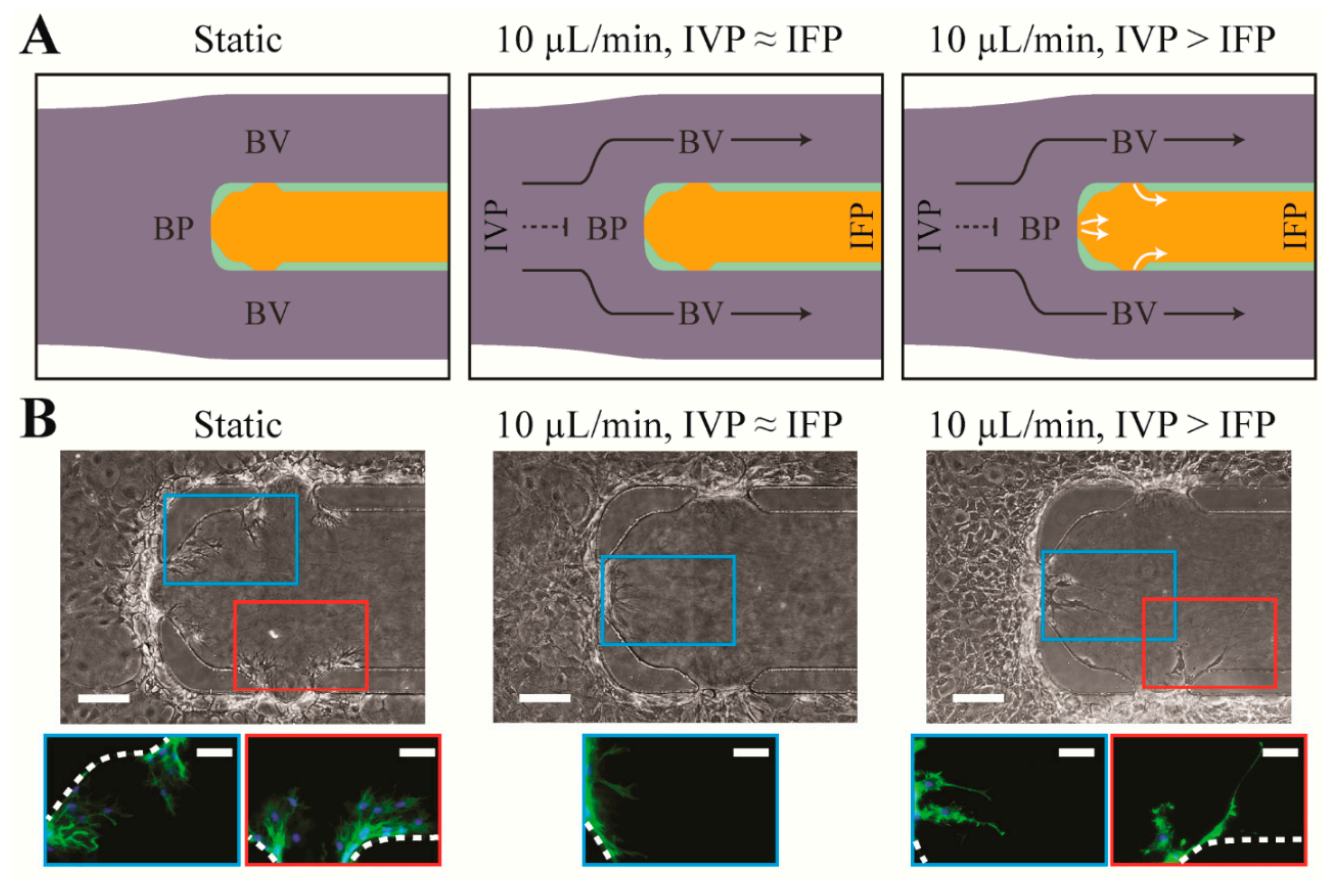

\section{C}

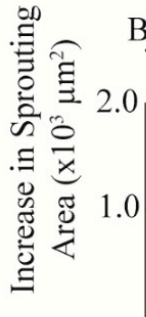

D

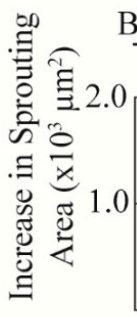

Perfusion Rate $(\mu \mathrm{L} / \mathrm{min})$ $\operatorname{BFF}\left(\mathrm{dyn} / \mathrm{cm}^{2}\right)$ :

$\operatorname{LSS}\left(\mathrm{dyn} / \mathrm{cm}^{2}\right)$

$\operatorname{TVF}(\mu \mathrm{m} / \mathrm{s})$ :

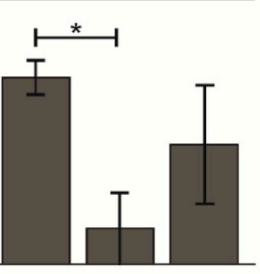

$\begin{array}{ccc}0 & 10 & 10 \\ 0 & 38 & 38 \\ 0 & 0 & 0 \\ 0 & 0 & 1\end{array}$

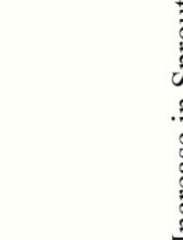

Perfusion Rate $(\mu \mathrm{L} / \mathrm{min})$ BFF

$\operatorname{LSS}\left(\right.$ dyn $\left./ \mathrm{cm}^{2}\right)$;

$\operatorname{TVF}(\mu \mathrm{m} / \mathrm{s})$ :
Branched Vessel (BV)

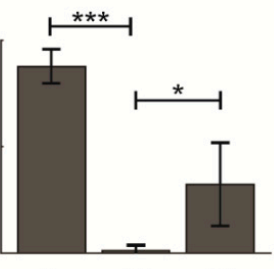

$\begin{array}{lll}0 & 10 & 10\end{array}$

$\begin{array}{lll}0 & 0 & 0\end{array}$

$\begin{array}{lll}0 & 3 & 3\end{array}$

$\begin{array}{lll}0 & 0 & 1\end{array}$

Figure 2. Application of bifurcating fluid flow (BFF) and laminar shear stress (LSS) attenuated endothelial sprouting. (A) The top-view schematics of the microdevice depicting the fluid mechanical factors under: (i) static, (ii) perfusion with equilibrated IVP and IFP resulting in BFF (dashed black line) at the BP, LSS (solid black line) in each BV and negligible transvascular flow (TVF), and (iii) perfusion with elevated IVP resulting in BFF at the BP, LSS in each BV and $1 \mu \mathrm{m} / \mathrm{s}$ TVF (white solid lines). (B) Representative phase contrast and epifluorescence images of sprouting MAECs in response to perfusion with $\sim 10 \mu \mathrm{L} / \mathrm{min}$ after $48 \mathrm{~h}$ under equilibrated and elevated IVP compared to static control condition. Dashed white lines mark the location of the polydimethylsiloxane (PDMS) posts. (C,D) Quantitative representation of the level of increase in sprouting area in response to treatment with each experimental test condition: at the BP, and in BV. *: p_value $<0.05 .{ }^{* * *}$ : $p_{-}$value $<0.001$.

The microfluidic devices were perfused with complete MAEC growth medium at a volumetric flow rate of $10 \mu \mathrm{L} / \mathrm{min}$ to generate $\sim 3 \mathrm{dyn} / \mathrm{cm}^{2}$ LSS in BV as previously described [14]. This LSS level is within the physiological range of shear stress in the microcirculation in vivo [23]. In addition, previously reported computational estimations showed that $10 \mu \mathrm{L} / \mathrm{min}$ perfusion flow rate in this microfluidic model generates $\sim 38 \mathrm{dyn} / \mathrm{cm}^{2}$ stagnation pressure at the BP imparting a normal force due to stagnation flow at the BP along with near-zero shear stress [14]. To our knowledge, there 
are no previous reports of direct measurements of stagnation pressure at microcirculatory vessel bifurcations in vivo. However, estimates for the bulk flow rate of a representative large arteriole in vivo are $\sim 2 \mu \mathrm{L} / \mathrm{min}$ based on the typical parameters for diameter $(60 \mu \mathrm{m})$ and mean velocity $(12 \mathrm{~mm} / \mathrm{s})$ [24]. Therefore, we estimate that the described level of stagnation pressure in this study $\left(\sim 38 \mathrm{dyn} / \mathrm{cm}^{2}\right) \mathrm{due}$ to the $10 \mu \mathrm{L} / \mathrm{min}$ perfusion rate and the geometrical characteristics of the utilized microfluidic model to be within the physiological range of BFF experienced in the microcirculation in vivo.

Application of $\sim 38 \mathrm{dyn} / \mathrm{cm}^{2} \mathrm{BFF}$ for $48 \mathrm{~h}$, in the absence of TVF, significantly attenuated endothelial sprouting area by $\sim 80 \%$ compared to the static control condition where MAECs undergo spontaneous sprouting (Figure 2B,C). Similarly, treatment with $\sim 3 \mathrm{dyn} / \mathrm{cm}^{2}$ LSS for $48 \mathrm{~h}$, in the absence of TVF, completely inhibited sprouting area (i.e., reduced by $\sim 98 \%$ compared to the static control condition) (Figure 2B,D). The observed suppression of MAEC sprouts by $\sim 3 \mathrm{dyn} / \mathrm{cm}^{2} \mathrm{LSS}$ is in line with previously reported results in vitro with human umbilical vein endothelial cells (HUVECs) in response to approximately the same LSS levels [11]. However, to our knowledge, the findings here are the first report that endothelial sprouting is attenuated by BFF.

\subsection{TVF Competes with BFF and LSS to Induce Formation of Angiogenic Sprouts}

After establishing the effects of BFF and LSS on sprouting, the effects of systematically introducing TVF $(\sim 1 \mu \mathrm{m} / \mathrm{s})$ via a static pressure head $(\sim 147 \mathrm{~Pa})$ along with the simultaneous fixed flow rate of $10 \mu \mathrm{L} / \mathrm{min}$ that produces $\sim 38 \mathrm{dyn} / \mathrm{cm}^{2} \mathrm{BFF}$ and $3 \mathrm{dyn} / \mathrm{cm}^{2}$ LSS in our bifurcation model was evaluated. Co-application of TVF with BFF at the BP resulted in a $~ 3$-fold increase in MAEC sprouting area compared to BFF alone, although this response was not statistically significant (Figure 2B,C). Similarly, co-application of TVF with LSS at the BV regions resulted in a 28 -fold increase in MAEC sprouting area compared to LSS alone (Figure 2B,D). The observed competing effects between TVF versus BFF and LSS on sprouting is in accordance with previous reports on the pro-angiogenic effect of TVF [11,12]. Furthermore, the level of endothelial sprouting induced by co-application of TVF alongside BFF and LSS was lower compared to the level of sprouting under static control condition. These findings suggest that the observed stabilizing effects of $38 \mathrm{dyn} / \mathrm{cm}^{2} \mathrm{BFF}$ and $3 \mathrm{dyn} / \mathrm{cm}^{2} \mathrm{LSS}$ counteracts the vessel sprouting responses triggered by a static pressure head of $\sim 147 \mathrm{~Pa}$ inducing a TVF of $\sim 1 \mu \mathrm{m} / \mathrm{s}$.

\subsection{Interstitial Flow Streamlines Coordinate Elongation of the Angiogenic Sprouts}

After establishing the effects of BFF, LSS, and TVF in triggering or suppressing endothelial sprouting, the role for interstitial flow through the 3-D ECM compartment due to IVP > IFP (Figure 2A) supported the alignment and elongation of MAEC sprouts was evaluated. It is known that endothelial cells cultured in 2-D (two-dimensional) configurations align and elongate with the direction of LSS within $24 \mathrm{~h}$ [25]. In addition, it was previously shown that when exposed to interstitial flow, breast cancer cells aligned parallel to flow streamlines [26]. The MAEC sprouts that formed in response to co-application of TVF alongside BFF and LSS at the BP and each BV, respectively, were more elongated (estimated average elongation index of $\sim 0.71$ ) compared to static control condition (estimated average elongation index of $\sim 0.41$ ). These results suggest that interstitial flow through the ECM helps promote the extension of sprouting MAECs.

Next, it was examined whether the local interstitial streamlines help coordinate the direction of MAEC sprouting. Interstitial flow streamlines that were previously determined by computational modeling [14] were superimposed onto the optical microscopy images of MAEC sprouting into the 3-D ECM (Figure 3). Under an ideal or perfect alignment, the orientation of the MAEC sprouts would be $0^{\circ}$ with respect to the interstitial flow streamlines. At the BP, MAEC sprouts that formed by co-application of TVF and BFF aligned with the direction of the local interstitial flow streamlines as visually demonstrated based on the superimposed images of the sprouting MAECs and the computationally estimated BP interstitial flow streamlines (Figure 3A). Our analysis by measuring the orientation angle provided an estimated average of $\sim 7^{\circ}$, thereby suggesting a high level of influence of the interstitial flow on the sprout alignment. In contrast, MAEC sprouts at the BV and formed under 
co-application of LSS and TVF do not prominently align with the local interstitial flow streamlines (estimated average angle of orientation of $\sim 49^{\circ}$ ), as visually depicted based on the overlapped image of sprouting MAECs and the local BV interstitial flow (Figure 3A). These outcomes were expected because at the BP aperture, the direction of TVF and interstitial flow in the supporting ECM are approximately parallel. In contrast, at the BV, the direction of interstitial flow changes prominently within $\sim 50 \mu \mathrm{m}$ away from the aperture interfaces (Figure 3A). Therefore, the MAEC sprout(s) that extend from the $\mathrm{BV}$ aperture interfaces are required to reorient in order to align with the direction of interstitial flow. Furthermore, it is noteworthy that the reported values for elongation index and alignment with interstitial flow serve as a descriptive assessment of the sprouting directionality. Thorough assessment of the role of interstitial flow in orchestrating sprouting directionality requires more advanced image analysis techniques that are beyond the scope of this study. Finally, it was confirmed that in the absence of MAECs, collagen matrix fiber orientation, i.e., the 3-D ECM, was not altered by perfusion and interstitial flow over $48 \mathrm{~h}$ (Figure 3B). Therefore, the observed alignment between angiogenic sprouts and the corresponding local interstitial flow streamlines is not mediated by flow-induced reorientation of the collagen fibers.

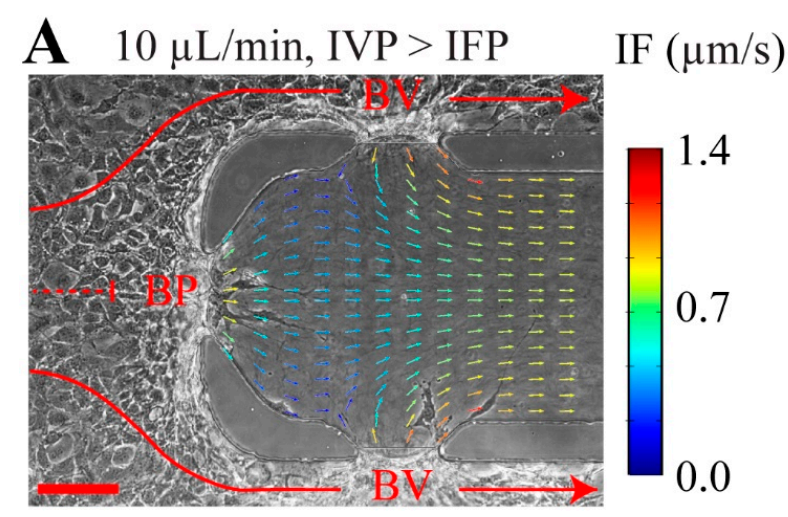

BP Aperture
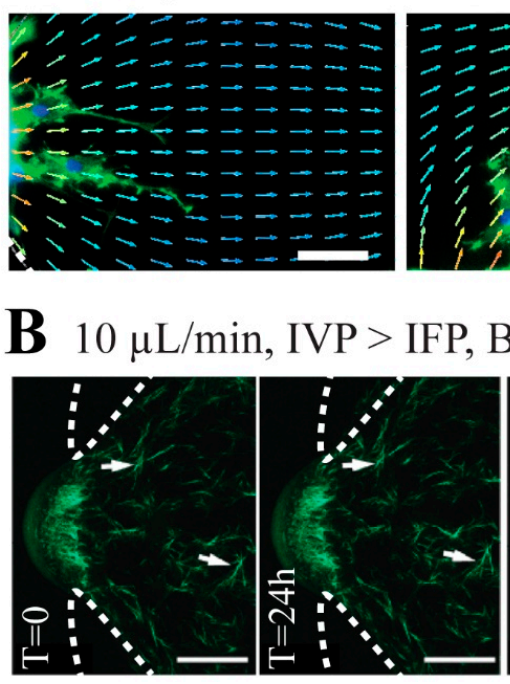

BV Aperture
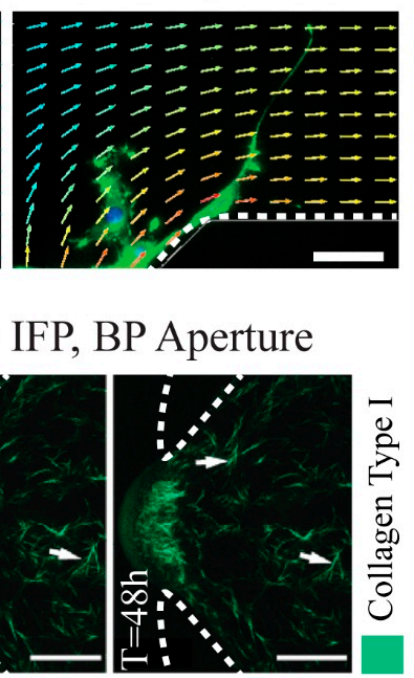

Figure 3. The angiogenic sprouts formed under elevated IVP were more elongated and showed alignment with the interstitial flow streamlines. (A) The computational interstitial flow streamline map superimposed on representative phase contrast and epifluorescence images of the angiogenic sprouts formed under elevated IVP condition. (B) Confocal reflectance microscopy images of collagen fibers under application of interstitial flow in the absence of MAECs. Arrowheads in the panels indicate landmark fibers to track among these images. Interstitial flow did not elicit a direct change in collagen fiber orientation. Red scale bar, $100 \mu \mathrm{m}$. White scale bars, $50 \mu \mathrm{m}$. Dashed white lines mark the location of the PDMS posts. 


\section{Discussion}

Obtaining a detailed and mechanistic understanding of the main physical regulators of angiogenic vascular sprouting and remodeling is significant for advancing therapeutic strategies for modulating pathological angiogenesis. These studies can be facilitated by biomimetic platforms that reconstitute tissue-level function in vitro while also enabling controlled application of pressure and shear stress to cultured endothelial cells. In this work, the effect of stagnation pressure at vessel bifurcations and LSS on sprouting angiogenesis in presence and absence of TVF was evaluated. The studies were conducted in an in vitro microfluidic device that presents BFF in the presence of a collagen ECM. The results reported here show, for the first time, that endothelial sprouting was inhibited at the bifurcation point at $38 \mathrm{dyn} / \mathrm{cm}^{2}$ stagnation pressure with nearly no shear stress. Application of $3 \mathrm{dyn} / \mathrm{cm}^{2} \mathrm{LSS}$ in the $\mathrm{BV}$ also imparted a stabilizing effect on the endothelium by suppressing the formation of angiogenic sprouts compared to static control conditions. While the LSS results agree with the previously reported role for tangential fluid forces in inhibiting VEGF induced endothelial sprouting [11], and decreasing endothelial permeability $[14,27]$ the inhibition of sprouting due to a stagnation pressure is a new finding. It is worth noting that past in vivo observations on increased endothelial sprouting from vessels with low to no blood flow, such as damaged or occluded vessels [28] or blind-ending sprouts [29], also corroborate the reported stabilizing effect of $3 \mathrm{dyn} / \mathrm{cm}^{2} \mathrm{LSS}$.

At the BP, the BFF represents negligible LSS but significant stagnation pressure arising from an impinging fluid flow. However, BFF did not show uncontrolled angiogenic sprouting. A previous in vivo study in the context of embryonic development showed that angiogenic sprouts form from points with local minimum shear stress except when the minimum shear occurs at the convergence of two blood vessels [30]. Interestingly, the fluid mechanical conditions at vessel convergences are comparable to flow characteristics at point of flow stagnation (locally stagnated flow with average negligible shear stress), which is based on the included angle of the incoming streams and the relative fluid shear of the converging fluid streams [31]. Furthermore, it was recently demonstrated that $38 \mathrm{dyn} / \mathrm{cm}^{2} \mathrm{BFF}$ at the BP for $6 \mathrm{~h}$ induced a significant decrease in endothelial permeability, thus causing stabilization of the endothelium [14]. In other words, as BFF stabilizes the endothelium, it is logical to expect a reduction in sprouting angiogenesis, which is the outcome we observed in this study.

Interestingly, simultaneous co-application of transvascular flow (TVF) alongside $38 \mathrm{dyn} / \mathrm{cm}^{2} \mathrm{BFF}$ and $3 \mathrm{dyn} / \mathrm{cm}^{2}$ LSS induced the formation of angiogenic sprouts at BP and in each BV, respectively. Therefore, while BFF and LSS stabilize the endothelium, the TVF competes with the effects of BFF and LSS to induce formation of angiogenic sprouts. The sprouts formed under co-application of TVF alongside BFF and LSS were more elongated compared to static control condition. Furthermore, the angiogenic sprouts formed at the BP under elevated IVP (i.e., presence of BFF and TVF) were visually observed to be along the direction of interstitial flow streamlines at the BP, but not at the BV (i.e., presence of LSS and TVF) after $48 \mathrm{~h}$. The visual alignment observations also find supporting evidence when taken together with the previous results on the pro-angiogenic effect of fluid flow across the endothelial monolayer [12,13].

Previous studies have attempted to discover the primary endothelial mechanosensory pathway that transduces the mechanical stimuli from LSS to the downstream endothelial biological response [32,33]. Since BFF is characterized by near-zero average shear stress at the stagnation point, it is plausible if the mechanotransduction pathway through which BFF leads to endothelial response is distinct from the previously reported pathways for LSS. Therefore, the detailed signaling pathways pertaining to BFF mechanotransduction requires further investigations. Moreover, while the results here were in the context of the blood vessel morphogenesis, it has previously been observed that intraluminal lymphatic valves preferentially form at the bifurcation points of collecting lymphatic vessels [34]. Our microfluidic model can be readily adapted to study the morphogenetic events coordinated by the local flow dynamics of branched vessels with applications for both the blood and lymphatic vasculature. 
In summary, the results reported here introduce BFF as a potent regulator of endothelial sprouting while showing that both bifurcating flows and tangential shear flows are important determinants of angiogenic sprouting in an in vitro microfluidic model of vessel bifurcations.

Author Contributions: Conceptualization, E.A., K.K.R., S.P., and J.W.S.; Methodology, E.A. and K.K.K.; Software, E.A.; Validation, E.A, K.K.R. and G.B.S.; Formal Analysis, E.A. and G.B.S.; Investigation, E.A. and G.B.S.; Resources, S.P. and J.W.S.; Data Curation, E.A. and G.B.S.; Writing-Original Draft Preparation, E.A. and G.B.S.; Writing-Review \& Editing, E.A., G.B.S., K.K.R., S.P. and J.W.S.; Visualization, E.A. and G.B.S.; Supervision, S.P. and J.W.S.; Project Administration, S.P. and J.W.S.; Funding Acquisition, S.P. and J.W.S.

Funding: This research was funded by The American Heart Association (15SDG25480000), NHLBI (R01HL141941), and Center for Emergent Materials, an NSF-MRSEC grant DMR-1420451. Images presented in this report were generated using the instruments and services at the Campus Microscopy and Imaging Facility, The Ohio State University. This facility is supported in part by grant P30 CA016058 from the NCI. Partial personnel support through the US Army Research Office through grant number W911NF-16-0278 and The Mark Foundation for Cancer Research (18-024-ASP) are also acknowledged. E.A. and K.K.R acknowledge OSU Presidential Fellowships. G.B.S. acknowledges funding from the Undergraduate Summer Research Program of The American Heart Association Great Rivers Affiliate, a Barry M. Goldwater Scholarship, and an Ohio State University (OSU) Comprehensive Cancer Center Pelotonia Fellowship.

Conflicts of Interest: The authors declare no conflicts of interest.

\section{References}

1. Pries, A.R.; Secomb, T.W. Making microvascular networks work: Angiogenesis, remodeling, and pruning. Physiology (Bethesda) 2014, 29, 446-455. [CrossRef]

2. Carmeliet, P.; Jain, R.K. Molecular mechanisms and clinical applications of angiogenesis. Nature 2011, 473, 298-307. [CrossRef]

3. Potente, M.; Gerhardt, H.; Carmeliet, P. Basic and therapeutic aspects of angiogenesis. Cell 2011, 146, 873-887. [CrossRef]

4. Greaves, N.S.; Ashcroft, K.J.; Baguneid, M.; Bayat, A. Current understanding of molecular and cellular mechanisms in fibroplasia and angiogenesis during acute wound healing. J. Derm. Sci. 2013, 72, $206-217$. [CrossRef]

5. Kerbel, R.S. Tumor angiogenesis. N. Engl. J. Med. 2008, 358, 2039-2049. [CrossRef]

6. Cao, Y. Angiogenesis modulates adipogenesis and obesity. J. Clin. Investig. 2007, 117, 2362-2368. [CrossRef]

7. Virmani, R.; Kolodgie, F.D.; Burke, A.P.; Finn, A.V.; Gold, H.K.; Tulenko, T.N.; Wrenn, S.P.; Narula, J. Atherosclerotic plaque progression and vulnerability to rupture: Angiogenesis as a source of intraplaque hemorrhage. Arter. Thromb. Vasc. Biol. 2005, 25, 2054-2061. [CrossRef]

8. Shibuya, M. Vascular endothelial growth factor and its receptor system: Physiological functions in angiogenesis and pathological roles in various diseases. J. Biochem. 2013, 153, 13-19. [CrossRef]

9. Akbari, E.; Spychalski, G.B.; Song, J.W. Microfluidic approaches to the study of angiogenesis and the microcirculation. Microcirculation 2017, 24, e12363. [CrossRef]

10. Wong, K.H.; Chan, J.M.; Kamm, R.D.; Tien, J. Microfluidic models of vascular functions. Annu. Rev. Biomed. Eng. 2012, 14, 205-230. [CrossRef]

11. Song, J.W.; Munn, L.L. Fluid forces control endothelial sprouting. Proc. Natl. Acad. Sci. USA 2011, 108, 15342-15347. [CrossRef]

12. Galie, P.A.; Nguyen, D.H.; Choi, C.K.; Cohen, D.M.; Janmey, P.A.; Chen, C.S. Fluid shear stress threshold regulates angiogenic sprouting. Proc. Natl. Acad. Sci. USA 2014, 111, 7968-7973. [CrossRef]

13. Vickerman, V.; Kamm, R.D. Mechanism of a flow-gated angiogenesis switch: Early signaling events at cell-matrix and cell-cell junctions. Integr. Biol. 2012, 4, 863-874. [CrossRef]

14. Akbari, E.; Spychalski, G.B.; Rangharajan, K.K.; Prakash, S.; Song, J.W. Flow dynamics control endothelial permeability in a microfluidic vessel bifurcation model. Lab Chip 2018, 18, 1084-1093. [CrossRef]

15. Nagy, J.A.; Dvorak, A.M.; Dvorak, H.F. Vascular hyperpermeability, angiogenesis, and stroma generation. Cold Spring Harb. Perspect. Med. 2012, 2, a006544. [CrossRef]

16. Huang, C.P.; Lu, J.; Seon, H.; Lee, A.P.; Flanagan, L.A.; Kim, H.Y.; Putnam, A.J.; Jeon, N.L. Engineering microscale cellular niches for three-dimensional multicellular co-cultures. Lab Chip 2009, 9, 1740-1748. [CrossRef] 
17. Srinivasan, R.; Zabuawala, T.; Huang, H.; Zhang, J.; Gulati, P.; Fernandez, S.; Karlo, J.C.; Landreth, G.E.; Leone, G.; Ostrowski, M.C. Erk1 and Erk2 regulate endothelial cell proliferation and migration during mouse embryonic angiogenesis. PLoS ONE 2009, 4, e8283. [CrossRef]

18. Song, J.W.; Gu, W.; Futai, N.; Warner, K.A.; Nor, J.E.; Takayama, S. Computer-controlled microcirculatory support system for endothelial cell culture and shearing. Anal. Chem. 2005, 77, 3993-3999. [CrossRef]

19. Boas, L.V.; Faustino, V.; Lima, R.; Miranda, J.M.; Minas, G.; Fernandes, C.S.V.; Catarino, S.O. Assessment of the Deformability and Velocity of Healthy and Artificially Impaired Red Blood Cells in Narrow Polydimethylsiloxane (PDMS) Microchannels. Micromachines (Basel) 2018, 9, 384. [CrossRef]

20. Rutkowski, J.M.; Swartz, M.A. A driving force for change: Interstitial flow as a morphoregulator. Trends Cell Biol. 2007, 17, 44-50. [CrossRef]

21. Chary, S.R.; Jain, R.K. Direct measurement of interstitial convection and diffusion of albumin in normal and neoplastic tissues by fluorescence photobleaching. Proc. Natl. Acad. Sci. USA 1989, 86, 5385-5389. [CrossRef]

22. Swartz, M.A.; Lund, A.W. Lymphatic and interstitial flow in the tumour microenvironment: Linking mechanobiology with immunity. Nat. Rev. Cancer 2012, 12, 210-219. [CrossRef]

23. Gray, K.M.; Stroka, K.M. Vascular endothelial cell mechanosensing: New insights gained from biomimetic microfluidic models. In Seminars in Cell \& Developmental Biology; Academic Press: Cambridge, MA, USA, 2017; pp. 106-117.

24. Popel, A.S.; Johnson, P.C. Microcirculation and Hemorheology. Annu. Rev. Fluid Mech. 2005, 37, 43-69. [CrossRef]

25. Levesque, M.J.; Nerem, R.M. The elongation and orientation of cultured endothelial cells in response to shear stress. J. Biomech. Eng. 1985, 107, 341-347. [CrossRef]

26. Polacheck, W.J.; Charest, J.L.; Kamm, R.D. Interstitial flow influences direction of tumor cell migration through competing mechanisms. Proc. Natl. Acad. Sci. USA 2011, 108, 11115-11120. [CrossRef]

27. Buchanan, C.F.; Verbridge, S.S.; Vlachos, P.P.; Rylander, M.N. Flow shear stress regulates endothelial barrier function and expression of angiogenic factors in a 3D microfluidic tumor vascular model. Cell Adhes. Migr. 2014, 8, 517-524. [CrossRef]

28. Yamada, H.; Yamada, E.; Hackett, S.F.; Ozaki, H.; Okamoto, N.; Campochiaro, P.A. Hyperoxia causes decreased expression of vascular endothelial growth factor and endothelial cell apoptosis in adult retina. J. Cell. Physiol. 1999, 179, 149-156. [CrossRef]

29. Anderson, C.R.; Hastings, N.E.; Blackman, B.R.; Price, R.J. Capillary sprout endothelial cells exhibit a CD36 low phenotype: Regulation by shear stress and vascular endothelial growth factor-induced mechanism for attenuating anti-proliferative thrombospondin-1 signaling. Am. J. Pathol. 2008, 173, 1220-1228. [CrossRef]

30. Ghaffari, S.; Leask, R.L.; Jones, E.A. Flow dynamics control the location of sprouting and direct elongation during developmental angiogenesis. Development 2015, 142, 4151-4157. [CrossRef]

31. Prakash, S.; Akberov, R.; Agonafer, D.; Armijo, A.D.; Shannon, M.A. Influence of Boundary Conditions on Sub-Millimeter Combustion. Energy Fuels 2009, 23, 3549-3557. [CrossRef]

32. Hahn, C.; Schwartz, M.A. Mechanotransduction in vascular physiology and atherogenesis. Nat. Rev. Mol. Cell Biol. 2009, 10, 53-62. [CrossRef]

33. Davies, P.F. Flow-mediated endothelial mechanotransduction. Physiol. Rev. 1995, 75, 519-560. [CrossRef]

34. Udan, R.S.; Dickinson, M.E. The ebb and flow of lymphatic valve formation. Dev. Cell 2012, 22, $242-243$. [CrossRef]

(C) 2019 by the authors. Licensee MDPI, Basel, Switzerland. This article is an open access article distributed under the terms and conditions of the Creative Commons Attribution (CC BY) license (http://creativecommons.org/licenses/by/4.0/). 